\title{
POP-ÄSTHETIK? \\ Überlegungen mit Blick auf Irmgard Keun und Christian Kracht
}

Von Maren Lickhardt (Innsbruck)

Der Artikel beschreibt Irmgard Keuns Romane `Gilgi - eine von uns` (I93I) und `Das kunstseidene Mädchen (1932) als Pop-Literatur. Es werden exemplarisch Parallelen zu Christian Krachts ,Faserland، (I995) gezeigt, die aus ähnlichen kulturellen Gemengelagen in den 2oer und 9oer Jahren resultieren. D. h. der Artikel arbeitet heraus, dass es Pop-Kultur bereits in den zwanziger Jahren gegeben hat und wie diese sich von der Populärkultur differenzieren lässt.

The article deals with Irmgard Keun’s `Gilgi - eine von uns` (I93I) and `Das kunstseidene Mädchen (I932) as pop-literature. It will show parallels with Christian Kracht's `Faserland (I995) that result from similar cultural conditions in the 20 s and 90s. So, the article will point out that there has already been something like pop-culture in the 20 s that differs from popular-culture.

\section{Einleitung}

Dass es sich bei Christian Krachts 'Faserland`) (1995) um einen Pop-Roman handelt, ist wohl kaum je in Abrede gestellt worden, was andere Zuordnungen, z.B. zum Bildungs- und zum Schelmenroman ${ }^{2}$ ) natürlich nie ausgeschlossen hat. Irmgard Keuns Romane , Gilgi - eine von uns $s^{3}$ ) (193I) und `Das kunstseidene Mädchen $\varsigma^{4}$ ) (1932) werden hingegen im Allgemeinen, wenn nicht als Zeitromane der Neuen Sachlichkeit, dann als Populärliteratur oder populäre Literatur der Weimarer Republik geführt. Diese Klassifikation sagt nicht mehr aus, als dass es sich um beliebte Texte handelt, was die Verkaufszahlen bezeugen, ${ }^{5}$ ) die

1) Christian Kracht, Faserland, Köln i995, S. I6. Im Folgenden zitiert mit der Sigle FL und Seitenangabe.

2) Niels Werber, Krachts Pikareske. Faserland neu gelesen, in: LiLi 44 (20I4), S. II9-I29.

3) Irmgard Keun, Gilgi - eine von uns, München 2003. Künftig zitiert mit der Sigle G und Seitenangabe.

${ }^{4}$ ) Irmgard Keun, Das kunstseidene Mädchen, hrsg. von Stefanie Arend und Ariane Martin, Berlin 2005. Künftig zitiert mit der Sigle KM und Seitenangabe.

5) Hiltrud Häntzschel, Irmgard Keun. Dargestellt von Hiltrud Häntzschel, Reinbek bei Hamburg 200I, S. 3 I. 
das allerdings auch für Thomas Manns Romane täten, und sie impliziert, dass es sich um einfache bzw. leicht zugängliche Romane handelt, was von niemandem bestätigt werden kann, der/die sich eingehender mit den Texten beschäftigt. ${ }^{6}$ )

Diese Zusammenstellung mag zunächst einmal nicht nur wie ein Vergleich von Äpfeln mit Birnen, sondern wie der von Äpfeln mit Bananen erscheinen. Aber liest man mit dem Lektürehorizont der Pop-Literatur der I990er Jahre bestimmte Romane der Weimarer Republik, müssen verblüffende und bislang nicht beachtete Parallelen auffallen. Das trifft insbesondere auf den einen oder anderen Text von Lili Grün, Ruth Landshoff-Yorck, Joe Lederer, Erich Maria Remarque, Walter Serner und Wilhelm Speyer $\left.\mathrm{zu}^{7}\right)$ - und auf Keuns Romane, die im Folgenden exemplarisch zur Debatte stehen sollen. Anhand einer wechselseitigen Perspektivierung von Keuns und Krachts Texten sollen Indizien für die Hypothese gesammelt werden, dass es Pop-Literatur in den zwanziger Jahren gegeben hat. - Nur nebenbei sei vermutet, dass es mehr Sinn ergibt, die Pop-Literatur der neunziger Jahre - auch als Pop II bezeichnet - vor diesem Hintergrund zu diskutieren, als sie stets mit der Pop-Literatur der sechziger Jahre - Pop I genannt - zu vergleichen und zu kontrastieren. ${ }^{8}$ )

Pop-Kultur - oder Pop - weist substantielle Unterschiede zum Populären und zur Populärkultur auf, deren Existenz in Bezug auf die zwanziger Jahre nicht in Frage steht und deren Untersuchung kein Desiderat darstellen würde. In einem ersten Schritt soll geklärt werden, worin der Unterschied zwischen Pop-Kultur, Populärem und Populärkultur besteht und welche Voraussetzungen in der Weimarer Republik gegeben sind, die es erlauben von Pop zu sprechen. In einem zweiten Schritt werden Keuns Romane mit kleinen Seitenblicken auf Krachts Text und unter Berücksichtigung einiger Forschungsergebnisse zur Pop-Literatur hinsichtlich der Merkmale beleuchtet, die sie als Pop-Romane erscheinen lassen. Bis zu dem Punkt bilden Pop-Konzepte der fünfziger bis neunziger Jahre den Ausgangspunkt, um Keuns Texte und die Kultur der Weimarer Republik in den Blick zu nehmen. Abschließend wird der Frage nachgegangen, ob Pop-Literatur mit einer spezifischen Pop- ̈̈sthetik einhergeht, die sich als textimmanente Signatur, Struktur oder Stil aufweisen lässt, und an diesem Punkt soll die Perspektive umgekehrt werden, können

${ }^{6}$ Kerstin Barndt, Sentiment und Sachlichkeit. Der Roman der neuen Frau in der Weimarer Republik, Köln 2003; - Maren Lickhardt, Irmgard Keuns Romane der Weimarer Republik als moderne Diskursromane, Heidelberg 2009; - Beate Kennedy, Zeit und Zitat. Narrative Verfahren und literarische Autorschaft im Gesamtwerk, Berlin 2014.

$\left.{ }^{7}\right)$ Ich plane eine Monografie, die sich des Themas mit Blick auf alle genannten AutorInnen annehmen wird und die 2019 erscheinen soll.

${ }^{8}$ ) Diedrich Diederichsen, Über Pop-Musik, Köln 20i4; - Christoph Rauen, Pop und Ironie. Popdiskurs und Popliteratur um I980 und 2000, Berlin 2010. 
Keuns Romane die Hintergrundfolie bilden, um das Konzept der Pop-Literatur wiederum mit Seitenblick auf Kracht zu hinterfragen.

\section{Populäres, Pop-Kultur, Populärkultur}

Was Pop im Sinne einer spezifischen Pop-Ästhetik in Bezug auf Literatur, was literarischer Pop, poppige Literatur oder Literatur-Pop sein könnte, ist noch nicht befriedigend geklärt, aber es gibt einen relativ stabilen, gut untersuchten Kanon dessen, was als Pop-Literatur bezeichnet wird. $\left.{ }^{9}\right)$ Berücksichtigt man die Korpusbildung sowie die häufigsten Annahmen zur neuen deutschen Pop-Literatur, ${ }^{10}$ ) kann festgehalten werden, dass sie sich - und das ist eine gleichermaßen banale wie wichtige Definition - als Reaktion auf Pop-Kultur konstituiert. $\left.{ }^{11}\right)$ Pop-Literatur ist also nicht ästhetisch, stilistisch, strukturell in anderen Hinsichten einheitlich definiert, sondern der gemeinsame Nenner ist die Bezugnahme auf Pop-Kultur. Das wirft die Frage auf, wie sich Pop-Kultur, Populäres und Populärkultur voneinander unterscheiden. ${ }^{12}$ )

9) Vgl. u.a. als historischen Längsschnitt: Moritz Bassler, Der deutsche Pop-Roman. Die neuen Archivisten, München 2002; - Thomas Jung, Vom Pop international zur Tristesse Royal: Die Popliteratur, der Kommerz und die postmoderne Beliebigkeit, in: Alles nur Pop? Anmerkungen zur populären und Pop-Literatur seit 1990, hrsg. von Thomas Jung, Frankfurt/M. 2002, S. 29-53; - Jörgen SchäfER (Hrsg), Pop-Literatur. (= Text + Kritik, Sonderband), München 2003; - Ecкhard Schumacher, Gerade Eben Jetzt. Schreibweisen der Gegenwart, Frankfurt/M. 2003; - Johannes G. Pankau (Hrsg.), Pop-Pop-Populär. Popliteratur und Jugendkultur, Bremen u. a. 2004; - Sascha SeIler, „Das einfache wahre Abschreiben der Welt". Pop-Diskurse in der deutschen Literatur nach 1960, Göttingen 2006; - Alexandra Tacke und Björn Weyand (Hrsg.), Depressive Dandys. Spielformen der Dekadenz in der Pop-Moderne (= Literatur, Kultur, Geschlecht 26). Köln, Weimar, Wien 2009. - Benjamin von Stuckrad-Barre favorisiert dagegen den Begriff, Literatur-Pop. (Interview, in: Pop seit 1964, hrsg. Eckhard Schumacher und Kerstin Gleba, Köln 2007, S. 365-399, hier: S. 368; Bassler, Der deutsche Pop-Roman (zit. Anm. 9), S. ioi).

${ }^{10}$ ) Im Wesentlichen bezieht sich dies auf die Pop-Literatur ab den 9oer Jahren, auch wenn diese selbstverständlich komplexer ist, und auch wenn es kontingent ist, hier den Vergleichspunkt anzusetzen, denn auch das Gerade, Eben, Jetzt der früheren Pop-Literatur (Schumacher, Gerade, eben, jetzt, zit. Anm. 9) ließe sich mit Keuns Text in Bezug setzen.

${ }^{11}$ ) Z.B. Thomas Hecken, Pop. Geschichte eines Konzepts. 1955-2009 (= Kultur- und Medientheorie), Bielefeld 2009, S. 465; Jung, Vom Pop international zu Tristesse Royale (zit. Anm. 9); - Jörgen SchäFer, „Mit dem Vorhandenen etwas Anderes als das Intendierte machen". Rolf Dieter Brinkmanns poetologische Überlegungen zur Pop-Literatur, in: SCHÄFER (zit. Anm. 9), S. 69-80, hier: S. 78. - Anders bei Dirk Frank, „Literatur aus den reichen Ländern." Ein Rückblick auf die Popliteratur der I990er Jahre, in: Poetik der Oberfläche. Die deutschsprachige Popliteratur der I990er Jahre, hrsg. von Olaf Grabienski, Till Huber und Jan-Noel Thon, Berlin, Boston 20II, S. 27-52, hier: S. 3 I.

${ }^{12}$ ) Niels Werber, Vortrag auf der Tagung 'Szenarien der Ausnahmer an der Universität Siegen, I7.-19.09.2015; Niels Werber, Vortrag im Rahmen der Ringvorlesung der Forschungsstelle >Populäre Kulturen an der Universität Siegen im Wintersemester 2015/16. Ich danke Niels Werber für die Bereitstellung der Manuskripte. Eine Zusammenfassung der Vorträge ist erschienen in: LiLi I83 (2016): Szenarien der Ausnahme in der Populärkultur. 
Das Populäre ist das, was viele beachten, ${ }^{13}$ ) was voraussetzt, dass die Massenmedien ein Apriori des Populären bilden..$^{14}$ ) Pop-Kulturen konstituieren sich nun unter Bezugnahme auf das Populäre auf Basis sichtbarer Stilverbünde. Sie haben die spezifische Funktion, das Populäre ästhetisch zu differenzieren. ${ }^{15}$ ) In dieser Konstellation unterhält Pop-Kultur eine parasitäre Beziehung zum Populären und transformiert es zu einer Frage des Stils. ${ }^{16}$ ) Bei Pop-Kulturen handelt es sich um stilistisch definierte Phänomene, die eine Unterscheidung zulassen und Identitäten anzeigen. Sie stellen Gruppen dar, die statt auf sozialen auf ästhetischen Variablen basieren. Das kontingente, gleich-gültige moderne Individuum bedarf dieser Formen, weil es nicht mehr selbstverständlich kulturell verortet ist. Pop-Kulturen führen also zu einer losen Kopplung von Individuen und reduzieren in ihrer sichtbaren Distinktionsfunktion Kontingenz in einer nicht mehr segmentierten oder stratifizierten modernen Gesellschaft. ${ }^{17}$ ) Die Zugehörigkeit zu einer Pop-Kultur, die mit einem Stilverbund angezeigt wird, ist insofern Ausdruck von Kontingenz, als sie aus einer freien Wahl resultiert, die auf einem Geschmacksurteil basiert und auf ganzer Linie ge- und erlebt wird. Dadurch erscheint Pop-Kultur als unmittelbar zugänglich und präsent, sodass sie dem hermeneutischen Nachgraben entgeht. ${ }^{18}$ )

Die pop-kulturelle Geschmackskultur wäre demnach ein Oberflächenphänomen, das unmittelbar sinnlich erlebt werden kann, aber man muss an dieser Stelle betonen, dass die En- und Decodierungsleistungen, die das Spiel mit der Distinktion erfordert, im Grunde doch Verstehensprozessen entsprechen, die sich nicht völlig inkommensurabel zu einer hermeneutischen Exegese hochkultureller Artefakte verhalten. Pop-kulturelle Stilverbünde bewegen sich in jedem Fall auf der Stelle, an der populäre Kulturen eine untrennbare Wechselwirkung mit jeweils ganz bestimmten Ästhetiken eingehen, die Grenzen zwischen Leben und Kunst verschwimmen, Lebensentwürfe in aisthetisch wahrnehmbaren Zeichenträgern encodiert sind. Dieser entdifferenzierte, avantgardistische Zug

${ }^{13}$ ) Thomas Hecken, Pop-Literatur um 1968, in: Schäfer, Text + Kritik. Sonderband PopLiteratur (zit. Anm. 9), S. 4I-54, hier: S. 4I.

$\left.{ }^{14}\right)$ Rudolf Helmstetter, Der Geschmack der Gesellschaft. Die Massenmedien als Apriori des Populären, in: Das Populäre der Gesellschaft. Systemtheorie und Populärkultur, hrsg. von Christian Huck und Carsten Zorn, Wiesbaden 2007, S. 44-72, hier: S. 44.

${ }^{15}$ ) Jochen Venus, Die Erfahrung des Populären. Perspektiven einer kritischen Phänomenologie, in: Performativität und Medialität populärer Kulturen. Theorien, Ästhetiken, Praktiken, hrsg. von Marcus S. Kleiner und Thomas Wilke, Wiesbaden 20I3, S. 49-74, hier: S. 54; - Thomas Hecken, Pop-Konzepte der Gegenwart. S. 96-99: <https://www. uni-muenster.de/Ejournals/index.php/pop/article/viewFile/755/720> [18.07.2018]; - THOMAS Jung, Trash, Cash oder Chaos? Populäre deutschsprachige Literatur seit der Wende und die sogenannte Popliteratur, in: Jung, Alles nur Pop? (zit. Anm. 9) S. I5-29, hier: S. 26.

${ }^{16)}$ Werber, Szenarien der Ausnahme in der Populärkultur (zit. Anm. I2).

$\left.{ }^{17}\right)$ Venus, Die Erfahrung des Populären (zit. Anm. I5), S. 54.

${ }^{18)}$ Ebenda, S. 56-57. 
von Pop-Kultur macht es schwer, einen kulturellen und einen ästhetischen Anteil eigens herauszupräparieren und zu beobachten. Wesentlich ist also, dass es sich bei Pop-Kulturen nicht lediglich um soziale, soziodemographisch oder sozialpraktisch konturierte Gebilde handelt, die hinsichtlich ihrer Mitgliederzahl eine kritische Masse erreicht haben, sondern dass im Lifestyle aisthetisch wird, also sinnlich wahrnehmbar, was diese Gebilde ausmacht. „Pop wäre demnach eine Form des Populären. ${ }^{\text {"19) }}$

Dadurch unterscheidet sich Pop von Populärkultur. Populärkultur ist ebenso wie Pop-Kultur - eine an einen gewissen Wohlstand und ein massenmediales Dispositiv gebundene Freizeit- und Unterhaltungskultur. ${ }^{20}$ ) Teilweise wird sie in der Forschung hinsichtlich der folgenden Merkmale nicht von Pop-Kultur unterschieden, ${ }^{21}$ ) jedoch rechtfertigen die Unterschiede auch eine Differenzierung in der Begriffsverwendung. Pop-Kulturen stellen, wie bereits gesagt, keine klassische soziale Kategorie dar, sondern eine sozio-ästhetische. Sie entstehen erst in dem Moment, in dem es zu einer durchgreifenden Entdifferenzierung von Gebrauchs- und Konsumgegenständen, Alltag und Kunst kommt, also - so gängige Forschungsmeinungen - in den fünfziger Jahren.

Mit der prägnanten postmodernen - oder vielleicht sollte man eher sagen: pop-modernen - Formel ,Cross the Border, Close the Gap ${ }^{22}$ ) sagt Leslie Fiedler beispielsweise dem bildungsbürgerlichen Kanon den Kampf an, um Artefakte der Massenkultur aufzuwerten und gleichberechtigt neben die Erzeugnisse der Hochkultur zu stellen. Dass es dabei auch um Rezeptionshaltungen und Wertungsperspektiven geht und nicht nur um produktions- oder werkästhetische Aspekte, wird im Titel eines Nachrufs auf Fiedler auf den Punkt gebracht: „Konsumiert die Kunst.“" $\left.{ }^{23}\right)$ Theoretische Positionen, die sich affirmativ auf den Konsum von künstlerischen und kulturellen Produkten aller Art beziehen, finden sich vermehrt ab den $50 e r$ Jahren, und zwar vorwiegend in den USA. ${ }^{24}$ ) Postmoderne und Pop sind die zwei neuen Begriffe, die teilweise zusammenhängend, teilweise unabhängig voneinander kursieren - und das so erfolgreich, dass es heute seltsam anmutet, in einem Aufsatz eine Vokabel wie Hochkultur

${ }^{19}$ Werber, Szenarien der Ausnahme in der Populärkultur (zit. Anm. I2).

${ }^{20}$ ) Hans-Otto Hügel, Einleitung, in: Hans Otтo-Hügel (Hrsg.), Handbuch Populäre Kultur. Begriffe, Theorien, Diskussionen. Stuttgart, Weimar 2003, S. $5 / 6$.

${ }^{21)}$ Ebenda, S. I I Iff.

${ }^{22}$ Leslie Fiedler, Cross the Border, Close the Gap, in: Playboy, Dezember 1969, S. I5 I, 230, $252-258$.

23) Christiane Zschirnt, Konsumiert die Kunst, in: taz, 8. Februar 2003, <http://www.taz. $\mathrm{de} / \mathrm{I} / \mathrm{archiv} /$ ?dig=2003/02/08/ao128> [18.07.2018].

${ }^{24}$ ) Thomas Hecken, Pop. (zit. Anm. II), S. 67. Spätestens in Bezug auf die Popliteratur der 9oer Jahre ist die Grenzverwischung von Hoch- und Populärkultur etabliert (Thomas ERnst, Popliteratur (= eva wissen), Hamburg 200I, S. 9). 
noch zu verwenden. Auf der anderen Seite entsteht die Pop Art, die von Seiten der Kunst eine Entdifferenzierung von Konsumkultur und Kunst betreibt. Natürlich sind dies nur wenige Aspekte in einem vielschichtigen Prozess, den Thomas Hecken gründlich aufgearbeitet hat. ${ }^{25}$ ) Aber eigentlich verliert Pop das ,Uläre' an dem Punkt, an dem es nicht nur zu einer Aufwertung der Massenund Populärkultur kommt, sondern umgekehrt die Bildende Kunst im Dienste ästhetischer Innovationen die Alltagskultur integriert und umgekehrt der Alltag ästhetisch aufgeladen wird. Indem sich letzteres vollzieht, entwickeln sich verschiedene ästhetische Stile, die als solche eine definitorische Funktion haben.

Statt der in der Populärkultur des 19. Jahrhunderts relevanten Frage „Sage mir, womit Du Dich unterhälst; und ich sage Dir, was Du bist. ${ }^{\left({ }^{26}\right)}$ könnte die Formel lauten: Zeige mir, was Du konsumierst, und ich sage Dir, wer Du bist. Demnach wäre, stark vereinfacht gesagt, ein Physiker Vertreter einer sozialen oder funktionalen Schicht oder eines Berufs, Schach- oder Computerspiele zu spielen, ein Aspekt der Populärkultur, in Kostümen auf Star-Trek-Conventions zu gehen, ein Phänomen der Pop-Kultur. ${ }^{27}$ ) Oder auf die Weimarer Republik bezogen, wäre die weibliche Angestellte eine Vertreterin einer neuen sozialen oder funktionalen Schicht oder eines Berufs, weibliches Mitglied in einem Sportverein zu sein, ein Aspekt der Populärkultur, die Neue Frau der zoer Jahre mit Bubikopf und Hüftnaht ein Phänomen der Pop-Kultur.

Dass jede Form der Unterhaltung auch aisthetisch gefasst ist und entsprechend analysiert werden $k a n n,{ }^{28}$ ) wird nicht in Abrede gestellt, aber Pop-Kultur ist im Gegensatz zu Populärkultur eine des reflexiven Stilbewusstseins. Selbst wenn man die Begriffe nicht differenzieren möchte, verhält es sich mit Blick auf Pop-Literatur ohnehin ganz einfach: Pop-Literatur ist keine Literatur, die sich auf Unterhaltungsphänomene im Allgemeinen bezieht. Für die Klassifikation als Pop-Literatur reicht also nicht, wenn - um ein Beispiel aus den 3oer Jahren einzuwerfen - eine junge Protagonistin wie Marieluise Fleißers >Mehlreisende Frieda Geier (193I) modernen Freizeitaktivitäten nachgeht und neue populäre Rollenbilder zu leben versucht. Sondern bei Pop-Literatur handelt es sich um Literatur, welche die reflexiv-ästhetischen Signale ihrer Kultur nutzt und sich als ihr Teil inszeniert.

Da viele Aspekte, die spezifisch für Pop konstitutiv sind, eine Entdifferenzierung von Kunst und Alltag voraussetzen, wie sie beispielsweise mit der Pop

25) Vgl. insg. Hecken, Pop (zit. Anm. ir).

${ }^{26)}$ Hügel, Handbuch Populäre Kultur (zit. Anm. 20), S. I8.

${ }^{27}$ ) Maren Lickhardt, Star Trek. Popkultur als Szene gemeinsamer Aufmerksamkeit. 2015 In: <http://www.pop-zeitschrift.de/2015/01/03/star-trekpopkultur-als-szene-gemeinsameraufmerksamkeitvon-maren-lickhardt3-I-2015/> [I8.07.2018].

${ }^{28)}$ HüGel, Handbuch Populäre Kulturen (zit. Anm. 20), S. i7/ı8. 
Art verfolgt wurde, bilden die fünfziger Jahre eine Zäsur. Aber auch die junge Kultur der Weimarer Republik hatte pop-kulturelle Züge, die pop-literarische Projekte angeregt haben - und die Rede ist im Folgenden nicht vom Dadaismus. Insbesondere durch den enormen Anstieg massenmedialer Angebote gerät das Populäre schon in den zwanziger Jahren zu einer schwer hintergehbaren Bedingung kultureller Selbstverständigung oder Selbstvergewisserung. Konkret spielen Lifestyle-Zeitschriften wie 'Uhu,, 'Dame und Querschnitt, 'Scherl's Magazinı, 'Elegante Welt und 'Sport im Bild eine große Rolle. In dieser dichten Medienkultur - und das ist so in der Forschung bislang nicht fokussiert worden - findet eine Transformation des Populären zu einer ästhetischen Kategorie statt und zwar in einem reziproken zirkulären Prozess zwischen Massenmedien, Kunst und alltäglichem ,Life-Style`.

Einige literarische Texte erhalten vor dem Hintergrund der virulenten Presse- und Kinolandschaft nicht nur eine intermediale bzw. interdiskursive Gestalt, sondern es kommt zu einer ästhetischen Inklusion von Konsumkultur und Warenästhetik aus der Werbung. Formal schlägt sich dies in einem paradigmatischen, oberflächigen Stil nieder. Funktional wird durch die implementierte Beachtungs- und Aufforderungssignatur von Massenmedien, Reklame etc. ein besonders wirksamer Appell an LeserInnen gerichtet, die eigene Lebenswelt ebenso zu ästhetisieren. Alltägliches aus der Konsumkultur findet nicht nur Eingang in die Kunst und verändert deren Formen, sondern das ästhetisch Aufgeladene wird wieder in den (Konsum-) Alltag zurückgespielt. Durch diese mehrfache Rückkopplung von Produktion und Rezeption stellt sich eine forciertere Ästhetisierung der Lebenswelt ein, durch die sich ein neues Distinktionsregime ausbildet, das eine wesentliche Sinnressource im Komplexitätsmanagement der Nachkriegszeit liefert.

Der Erste Weltkrieg hatte - es mutet etwas banal an, diesen Satz so zu schreiben - auch eine epistemologische und axiologische Lücke gerissen. Nicht nur eine existentielle Erschütterung ist in den zwanziger Jahren spürbar, sondern die alte Ordnung ist obsolet geworden, insbesondere ihre gesellschaftlichen Klassifikations- und Distinktionsschemata. ${ }^{29}$ ) Dafür bieten sich aber im Rahmen der relativen, wirtschaftlichen Stabilisierungsphase ab 1924 Raum und Kapazitäten für Massenproduktion, Massenkonsum und Freizeitkultur, ${ }^{30}$ ) die das Rohmaterial für pop-kulturelle Stildistinktionen bereit stellen. Der Zu-

${ }^{29)}$ Helmut Lethen, Verhaltenslehren der Kälte. Lebensversuche zwischen den Kriegen, Frankfurt/M. 1994, S. IO-I I, 36.

30) Detlev Peukert, Die Weimarer Republik. Krisenjahre der Klassischen Moderne, Frankfurt/M. I987, S. I77-I78; - Peter Hoeres, Die Kultur von Weimar. Durchbruch der Moderne, Berlin-Brandenburg 2008, S. I05, I09; - Ursula BüTtNer, Weimar. Die überforderte Republik I918-1933. Leistung und Versagen in Staat, Gesellschaft, Wirtschaft und Kultur, Stuttgart 2008, S. 33 I. 
sammenbruch der alten sozialen Ordnung setzt die Individuen frei, löst sie auf und führt sie - zumindest gefühlt - der Vermassung zu. Durch Massenmedien und Massenproduktion stehen Ressourcen zur Verfügung, anhand derer sich neue Identitäten und Differenzen entfalten, sich das Individuum wieder als Teil einer sichtbar definierten Gruppe konstituiert, sich die Masse also ästhetisch formt. Dabei handelt es sich um Entwürfe, die in die Zukunft gerichtet sind, die auflösen, was es an essentialistischen Restzuschreibungen noch gibt. Insofern ist Pop eine Angelegenheit, die sich nicht mit dem aufkommenden Nationalsozialismus verträgt und von diesem mit biologistischen Konzepten bald unerbittlich zurückgedrängt werden sollte.

\section{Irmgard Keuns Romane der Weimarer Republik als Pop-Romane?}

Dass im Kontext von Pop die Unterscheidung in highbrow und lowbrow literature dekonstruiert wird, dass die Oberfläche aufgewertet und die lustvolle Rezeption bzw. das Konzept der Unterhaltung gutgeheißen wird, sind Facetten eines Prozesses, der in den neunziger Jahren so weit fortgeschritten ist, dass sich Christian Kracht nicht mehr fundamental am Verdacht der Trivialität abarbeiten muss. Irmgard Keun dagegen führt in ihren Romanen eine reflexive poetologische Verortung ihrer eigenen Ästhetik mit. Ganz im Sinne Fiedlers nimmt Keun eine Relativierung von Kunst-Rezeption und -Bewertung vor. In , Gilgi< wird beispielsweise ein Konzertbesuch der Protagonistin zum Anlass genommen, einen sinnlich unmittelbaren und einen intellektuell gebildeten Zugang zu derselben Musik einander gegenüber zu stellen. Dabei wird keine Umkehrung des traditionellen hochkulturellen Wertungsschemas vorgenommen, anhand dessen ein kulturelles und ästhetisches Gefälle auf Basis einer vermeintlichen Orientierung an Substanz und Qualität von Kunst postuliert wird. Vielmehr vollzieht sich eine gleich-gültige Betrachtung, indem Kunstbewertung im rezipierenden Subjekt verankert wird. ${ }^{31}$ )

Sämtliche Kunstformen können zum Spielball relativistischer sinnlichästhetischer oder emotional-ästhetischer Umgangsweisen werden. „Was dem einen sein Huberman - bleibt dem andern sein Dajos Bela, was dem einen sein Rembrandt ist - ist dem andern sein Abeking. Was will man da machen?" (G I44/I45). Im «Kunstseidenen Mädchen` beschreibt die Ich-Erzählerin die „winzigen goldenen Fetzen“ im Danziger Goldwasser, die man zwar sehend ge-

${ }^{31}$ ) Dass die Musik in dieser Passage bei Gilgi körperliche Empfindungen hervorruft, soll nicht unerwähnt bleiben, weil der Topos der Musik als Medium der Unmittelbarkeit im Kontext der Pop-Literatur häufiger auftaucht. In Keuns Romanen spielt dies eine Rolle, allerdings eine untergeordnete, während anhand musikalischer Referenzen viel stärker und regelmäBiger der kulturelle Bewertungsdiskurs thematisiert wird. 
nießen, derer man aber nicht haptisch habhaft werden kann. Das wird nicht als Mangel ausgewiesen, denn „es ist hübsch, zu wissen, daß man Gold trinkt, das süß schmeckt, wovon man betrunken wird [...]“ (KM 77/78). Genau gelesen, macht hier nicht der - mit dem Markennamen benannte - Alkohol betrunken, sondern das Gold.

Doris fällt kulturkritischen Deutungsmustern zufolge den Illusionen der Kulturindustrie zum Opfer. ${ }^{32}$ ) Tatsächlich wird im Roman aber Sehen und Erleben der vermeintlichen Substanz vorgezogen.33) Der sinnliche Genuss betrügt nicht, sondern er hält nicht mehr und nicht weniger, als er verspricht. Im Text ist eine Genussfähigkeit für Rauschhaftigkeit angelegt, die nur den Augenblick kennt. Wer dies zu schätzen weiß, profitiert davon; wer weitersucht, dem wird nichts geboten. Der Roman tilgt hochkulturelle Implikationen einer vermeintlichen ontischen Präsenz, um in einem reflexiven Spiel die ästhetische Oberfläche als solche und deren subjektive, lustvolle Rezeption als Ausdruck eines spätmodernen Relativismus ohne metaphysische Hinterwelten zu radikalisieren. Dem Kunstwerk wird keine absolute Qualität zugeschrieben, sondern es geht um seinen Effekt in einem selbstgenügsamen und selbstreferentiellen wenn man so will, ,leeren', aber nicht lustlosen - Prozess. Wichtig ist gerade darum, dass die Lust auch geteilt wird. Insofern ist das von Keun beschriebene Kunstwerk weder autonom noch interesselos.

Und Therese ißt meine Pralinees und ist froh, daß ich wieder ein Erlebnis hatte. [...] Es macht mir furchtbar Spaß, ihr zu erzählen, weil sie eine unerhörte Art hat, sich zu verwundern [...] wenn ich ihr nicht erzählen könnte, hätte ich nicht so große Lust, fabelhafte Erlebnisse zu haben. (KM 19f.)

Nicht nur die Gewissheit, eine Zuhörerin zu haben, macht das Erzählen erzählenswert, sondern erst die Möglichkeit, davon zu erzählen, macht Erleben überhaupt erlebenswert. ${ }^{34}$ ) Die in Aussicht stehende Ästhetisierung motiviert das Leben, was - nebenbei bemerkt - kein ganz un-elitäres Unterfangen darstellt.

Aber die stilistisch erschaffene und poetologisch überhöhte Oberflächenästhetik geht in beiden Romanen eine Verbindung mit der Weimarer PopKonsum-Unterhaltungs-Kultur ein. Insofern erweist sich Keun als Vorläuferin der 'neuen Archivisten`, also als Avantgarde der Pop-Literatur. ${ }^{35}$ ) Ihre Romane

32) Liane Schüller, Vom Ernst der Zerstreuung. Schreibende Frauen am Ende der Weimarer Republik. Marieluise Fleißer, Irmgard Keun und Gabriele Tergit, Bielefeld 2005 , S. I62.

33) Heide Volkening, Karriere als Komet. Working Girls jenseits des Happy End, in: Working Girls. Zur Ökonomie von Liebe und Arbeit, hrsg. von Sabine Biebl, Verena Mund und Heide Volkening, Berlin 2007, S. 204-224, hier: S. 223.

34) Anne Fleig, Das Tagebuch als Glanz: Sehen und Schreiben in Irmgard Keuns Roman Das kunstseidene Mädchen, in: Irmgard Keun. Deutungen und Dokumente, hrsg. von Stefanie Arend und Ariane Martin, S. 45-6o, hier: S. 59.

${ }^{35}$ ) Bassler, Der deutsche Pop-Roman (zit. Anm. 9). 
setzen sich materiell in großem Maß aus fremden Versatzstücken zusammen, stellen populärkulturelle Paradigmen ihrer Zeit exzessiv aus, sodass sie nur auf den ersten Blick eine originelle, geschlossene Diegese entfalten. ${ }^{36}$ ) Neben vielem mehr finden sich in den Texten Markennamen, die in der Diskussion um die Pop-Literatur der 9oer Jahre eine so große Rolle gespielt haben. ${ }^{37}$ ) Indem die Romane diese in hoher Frequenz benennen (u. a. Elisabeth-Arden-Kosmetik (G 229), Coty-Kosmetik (KM 50/5I) etc.), funktionieren sie nur unter der Voraussetzung zeitgenössischer Massenmedien, in denen z.B. Waren- und Körperästhetik verschränkt werden. Das Anführen konkreter Markennamen bietet die Möglichkeit, mit nur einem Wort Erscheinungsbilder, kulturelle Praktiken, soziale Bedeutungen, gesellschaftliche Wertungen etc. zu evozieren, da all dies bereits außerliterarisch an dem einen Wort kristallisiert ist. $\left.{ }^{38}\right)$ Die Romane setzen auf Praktiken des Konsums und des Self-Fashioning, die sich längst zwischen Lifestyle-Zeitschriften und Rezipientinnen eingestellt haben, um sowohl eine ästhetische Oberfläche zu etablieren als auch Geschichten zu erzählen.

Keuns Texte gehen unmittelbar aus der Mitte der Warenwelt und Konsumkultur hervor. Dies wird als Bedingung ihrer selbst nicht mehr in Frage gestellt, sondern herausgestellt. ${ }^{39}$ ) Keun arbeitet sich nicht an künstlerischen avantgardistischen Entdifferenzierungen ab, sondern setzt bereits einen primär nichtkünstlerischen Bereich voraus, in dem dies stattgefunden hat, der allerdings mit einem Höchstmaß an Ästhetik aufgeladen ist: Lifestyle-Zeitschriften, Kino, Reklame etc. Dem entsprechend leben Keuns Romane von der Nennung von Liedtexten, Namen von Filmstars, Szenelokalen etc. Weite Teile des Textes bestehen aus Collagen aus Lifestyle-Zeitschriften, ${ }^{40}$ ) in denen pop-kulturelle Stil-Attitüden ausgehandelt werden. Auf das Collage-Verfahren wird wiederum poetologisch hingewiesen. Gilgis leibliche Mutter, also ihr körperlich-materieller Ursprung wird im Text wörtlich als „Magazindame“ (G 229) bezeichnet.

36) Gesche Blume, Irmgard Keun. Schreiben im Spiel mit der Moderne, Dresden 2005, S. I6; Gudrun RafF, Leben. Szenen eines Täuschungsspiels. Zu literarischen Techniken Irmgard Keuns, Diss. Hamburg 2000, S. 40-4I, I26.

37) Vgl. zu Marken in der Literatur allgemein Moritz Bassler, Moderne und Postmoderne. Über die Verdrängung der Kulturindustrie und die Rückkehr des Realismus als Phantastik, in: Literarische Moderne. Begriff und Phänomen, hrsg. von Sabina Becker und Helmuth Kiesel, Berlin, New York 2007, S. 435-450; - Björn Weyand, Poetik der Marke. Konsumkultur und literarische Verfahren 1900-2000, Berlin, Boston 2013.

${ }^{38}$ ) Bernd Seiler, Die leidigen Tatsachen. Von den Grenzen der Wahrscheinlichkeit in der deutschen Literatur seit dem I8. Jahrhundert, Stuttgart 1983, S. 262-263; - Gisela MüTING, Die Literatur bemächtigt sich der Reklame. Untersuchungen zur Verarbeitung von Werbung und werbendem Sprechen in literarischen Texten der Weimarer Zeit, Frankfurt/M. 2004, S. 248-249; - Bassler, Der deutsche Pop-Roman (zit. Anm. 9), S. I6I.

39) Lickhardt, Irmgard Keuns Romane (zit. Anm. 6).

${ }^{40)}$ Ebenda. 
Doris schreibt in ein Buch, das sie verziert, indem sie ausgeschnittene Tauben darauf klebt (KM I2). Was in den Text collagiert ist, was er als kulturelle Versatzstücke sammelt, sammelt Doris in der Fiktion auf ihren Streifzügen durch Berlin als sinnliche Eindrücke.

"Doris - ein Wald«, sagt er. Wald! - sind ja doch in Berlin. [...] Wenn er doch sprechen würde! - wir wollen weiter - manchmal kommt jetzt ein halber Stern - aber doch nichts gegen die Reklame - [...]. Berlin, ich zeige ihm doch Berlin. Und im Vaterland toll elegante Treppen wie ein Schloß mit Gräfinnen, die schreiten - und Landschaften und fremde Länder und türkisch und Wien und Lauben von Wein und die kolossale Landschaft eines Rheines mit Naturschauspielen, denn sie machen einen Donner. (KM 110)

Natur wird geringgeschätzt; die urbane Konsumkultur aufgewertet. Ein Stern ist nichts gegen Reklame, und statt einem Wald bietet die Ich-Erzählerin ihrem - allerdings blinden - Freund die Dekoration der angesagten Berliner Gaststätte „Haus Vaterland“, das mit seinen künstlichen „Landschaften“ eine frühe Form der Erlebnisgastronomie inszenierte.

Wenn Pop-Literatur sich durch ihre diskursive Referenz auf Pop-Kultur konstituiert oder dieserart definiert wird, ${ }^{41}$ ) ist klar, dass das stilistisch-sichtbare Distinktionsmerkmal der Literatur vorgelagert ist. Es wird auf einen bereits präformierten Nexus zurückgegriffen. Pop-Literatur schöpft durch ihre Referenzen nicht nur aus dem Paradigmenvorrat der Pop-Kultur, ${ }^{42}$ ) sondern auch aus bereits ausgeprägten Paradigmenkombinationen. So passt KalodermaKosmetik zur jungen Gilgi, während sich ihre mondäne leibliche Mutter mit Elisabeth-Arden pflegt, und das weiß man aus der Reklame in den zeitgenössischen Lifestyle-Zeitschriften. Bei den Referenzen handelt es sich nach allgemeiner Auffassung um Synekdochen in dem Sinn, dass sie pars pro toto für einen bestimmten Lebensstil stehen, der als Ganzes an diesen Teilen ablesbar ist. Die Literatur ist an diesem Punkt zunächst einmal weitgehend Medium als durchlässiges Zwischenglied. ${ }^{43}$ )

Eine spezifische ,poppige` Ästhetik lässt sich zunächst einmal nicht erkennen, d.h. man kann Literatur nur schwer eine unmittelbare Erlebnisqualität zuweisen oder in ihr einen sinnlichen Reiz sehen, was üblicherweise für Pop gel-

${ }^{41}$ ) Legt man probehalber einen spezifischeren Kriterienkatalog an (Frank, Literatur aus den reichen Ländern, zit. Anm. II, S. 32), kann dieser in Bezug auf Keuns Texte abgehakt werden. Es liegt bei Keun eine lebensweltliche Orientierung vor; es finden ästhetische Grenzüberschreitungen statt; popmusikalische oder audiovisuelle Strukturen spielen eine Rolle; es handelt sich um Zielgruppenliteratur; und die Autorin war Medienarbeiterin.

${ }_{42}$ Bassler, Der deutsche Pop-Roman (zit. Anm. 9) insgesamt und bes. S. IO2.

${ }^{43}$ ) Im Latourschen Sinne könnte man fragen, inwieweit sich das neutral funktionierende Zwischenglied zu einem Mittler mit einer gewissen Eigendynamik wandelt (BRUno LATOur, Eine neue Soziologie für eine neue Gesellschaft. Einführung in die Akteur-NetzwerkTheorie, Frankfurt/M. 2007, S. 70). 
tend gemacht wird. ${ }^{44}$ ) - Natürlich ließe sich das im Fall von Lautgedichten bestreiten, aber das Beispiel ist an der Stelle zu weit hergeholt. - Baßler hat darauf aufmerksam gemacht, dass Pop-Literatur als Paradigmen-Archiv fungiert, und in dem Kontext gezeigt, dass neue poetische Formen an diese Anlage geknüpft sind, die Roman Jakobsons Verständnis der poetischen Funktion der Sprache umkehren.$^{45}$ ) „Die poetische Funktion überträgt das Prinzip der Äquivalenz von der Achse der Selektion auf die Achse der Kombination. ${ }^{(46)}$ ) Gefragt wird also in Bezug auf Pop-Literatur nach ihrer Paradigmen-Selektion, nicht nach deren äquivalenter Kombination. Natürlich wäre es zu kurz gegriffen, diese lediglich als Stilmittel, Klangwirkungen etc. zu verstehen, aber dennoch implizieren Jakobsons Ausführungen, dass sich die poetische Funktion dadurch konstituiert, dass Kombinationen und damit auch Selektionen innersprachlich motiviert sind. Daraus ergibt sich auch die von Jakobson postulierte Autonomie der poetischen Funktion, der Selbstzweck der Mitteilung. ${ }^{47}$ ) Zu Pop will das nicht recht passen - sehr wohl aber Baßlers komplementäre Betrachtung. Pop-Literatur, die sich dadurch als solche konstituiert, dass sie aus einem popkulturellen Paradigma Bezugnahmen ostentativ herausstellt, ist nicht autonom. Vielmehr kommen in ihr ganz andere Sprachfunktionen forciert zum Tragen, z.B. neben der referentiellen Funktion die appellative Funktionen der Warenund Konsumästhetik.

Christian Krachts 'Faserland wurde neben anderen Romanen in dieser Hinsicht ausgiebig untersucht. Die konkret benannte Barbour-Jacke ist in der Forschung und im Feuilleton geradezu topisch geworden, und jeder weiß, auf welche Szene, welchen Lebensentwurf, welche Stilentscheidung, welchen Wertehorizont die Nennung dieser Marke rekurriert. Barbour wäre aber nach Jakobson nur dann eine ästhetische Entscheidung, wenn der Ich-Erzähler auch Bourbon trinken würde. Das tut er im Textverlauf zwar auch (FL 54). Im Kontext der ersten aufmerksamkeitserweckenden Nennung der Jackenmarke, und zwar auf den ersten beiden Seiten fünf Mal, wird aber Chablis getrunken, was auch gleich mehrere Male angemerkt wird. D.h. der Text verbleibt kombinatorisch auf der paradigmatischen Ebene, deren Äquivalenzen kulturell vorgegeben sind, und nutzt Versatzstücke nicht im Sinne Jakobsons zu einer äquivalenten syntagmatischen Kombination. Innerästhetisch motiviert oder

44) Hecken, Pop-Konzepte der Gegenwart (zit. Anm. I5).

${ }^{45}$ ) Bassler, Der deutsche Pop-Roman (zit. Anm. 9), S. io2; - Niels Werber, „Das graue Tuch der Langeweile“. Der Dandy als Motiv und Verfahren der Literatur 1900/2000, in: TaCke/Weyand, Depressive Dandys (zit. Anm. 9), S. 63, 70.

${ }^{46)}$ Roman Jakobson, Linguistik und Poetik, in: Roman Jakobson, Poetik. Ausgewählte Aufsätze 1921-I97I, Frankfurt/M. 1979, S. 83-I21, hier: S. 94.

${ }^{47)}$ Ebenda, S. S. 92-93 
,autonom' sind die Äquivalenzen der einzelnen Elemente nicht, sondern sie ergeben sich auf der referentiellen Ebene. - Nebenbei bemerkt, scheint Krachts Roman am wenigsten von allen üblicherweise dieser Kategorie zugeordneten Texte ein Pop-Roman zu sein, arbeitet er sich doch ganz traditionell am (Anti-)Bildungs- und dem Schelmenroman ab. - Aber natürlich verhält sich der Roman gegenüber pop-kulturellen Elementen keineswegs passiv. Vielmehr werden die Versatzstücke in den Texten bewertet. Es ist sehr bezeichnend, dass sich Krachts Ich-Erzähler bei jeder Gelegenheit übergibt, also all das auskotzt, womit er von der Warenwelt gefüttert und übersättig wird. ${ }^{4}$ ) Und auch andere Figuren in seinem Roman erbrechen sich als Zeichens des Einbruchs einer unkontrollierbaren Körperlichkeit, die als rhetorisches Mittel der Authentizität und somit gegen die im Roman verhandelten Diskurse eingesetzt wird.

[...] und dann dreht sie sich weg und übergibt sich in die Badewanne. Nicht so ein normales Übergeben, sondern ein richtiger Schwall, wie in Der Exorzist, nur eben nicht grün, sondern rot. Die Kotze klatscht in die Badewanne, und man kann es richtig sehen, was sie alles getrunken haben muss [...] und dazwischen sind noch ein paar Klümpchen irgendwelcher unverdauter Speisen, sieht aus wie Karotten und ein wenig Mais. (FL 42)

Die Übelkeit springt direkt von der Figur auf Lesende über, was eine ästhetische Leistung ist, aber eben nicht im Sinne des Pop-Diskurses.

Keuns Romane verhalten sich äußerst affirmativ gegenüber der Pop-Kultur. Die Collage-Texte existieren ostentativ unter deren Voraussetzung in einer dichten Medienkultur. ${ }^{49}$ ) Das ist die Bedingung ihrer Existenz und wird als solche prinzipiell nicht als Mangel ausgewiesen, sondern steht für neue Möglichkeiten, Lust und Selbstermächtigung. Das heißt aber nicht, dass die Texte überhaupt keine Kritik übten. Sie funktionieren nicht nur reflexiv in Bezug auf sich selbst, sondern spielen die Reflexionen durchaus kritisch zurück. So nutzt die Figur Gilgi die von Werbe- und Boulevardtexten vorgegebenen Sprachschablonen zur Beschreibung eines Automobildesigns, um das Bewegungsmuster ihrer Spucke anzukündigen.

Wart einen Augenblick, Martin - wie macht sich das: linker Fuß auf der Erde, rechter Fuß auf dem Trittbrett - Frau Generaldirektor X. mit ihrem schnittigen, rassigen, elegant karossierten 17/100-PS-Viersitzer-Cabriolet! Elegante Welt - letzte Seite... Entschuldige, Martin, aber das reizt mich, ordinär zu werden! Ich werd' jetzt im erstklassigen, schnittigen, elegant karossierten Dreimeterbogen bis zur Wand drüben spucken. (G 164)

${ }^{48}$ ) Dazu genauer: Carrie Smith-Prei, Kölner Realismus Redux. The Legacy of ig6os Realism in Postunification Literature, in: Closing Borders, Bridging Gaps? Deutscher Pop an der Jahrtausendwende (Special issue of ,Literatur für Leser), hrsg. von ANke BiendarRa, 3I (2008), S. 8I-93.

49) So auch die neue deutsche Pop-Literatur nach Margaret McCarthy, Introduction, in: German Pop Literature, hrsg, von Margaret McCarthy, Berlin, Boston 20I5, S. I-30, hier: S. 4. 
Die Pose der Neuen Frau auf dem Trittbrett eines Automobils ist Zeitschriftenlesern und -leserinnen der Weimarer Republik allzu vertraut, um nicht zu sagen bis zum Erbrechen vertraut. ${ }^{5 \circ}$ ) Gilgi//Gilgi` schluckt nicht alles, was ihr vorgegeben wird bzw. sie spielt es spiegelnd und imitierend zurück.

\section{Pop-Ästhetik?}

Über diese Bewertungen hinaus sind die in die Texte eingelassenen PopParadigmen natürlich stilbildend und ästhetisch. Wie Moritz Baßler zu Recht festgestellt hat, besteht der Clou von Pop-Literatur eigentlich darin, dass sie das Paradigma nicht im Syntagma abbildet, sondern es als Paradigma herausstellt. $\left.{ }^{\text {II }}\right)$ Eine derartige Kompilation ist strukturell relevant, weil sie Beschreibungen ersetzt, weil fremde Zusammenhänge unmittelbar zum Sprechen kommen, weil die Texte sich nicht mehr selbst erzählen, weil sie unabgeschlossen und offen werden, weil die Art und Weise der Zusammenstellung, die Einbettung, die Übergänge und deren Markierungen Unterschiede macht. In Bezug auf Baßler ist zu beachten, dass, wenn er vom Herausstellen des Paradigmas spricht, er nicht mehr vom Paradigma spricht, sondern vom Herausstellen, also einer poetischen Funktion. An dem Punkt kann den Texten eine Agency zugestanden werden, und es wäre zu verfolgen, wie die Texte das Paradigma herausstellen, also reflexiv, ostentativ, geordnet, ungeordnet usw. usf., nicht nur, um welches Paradigma es sich handelt. Es bleibt fraglich, ob man anhand der angerissenen Verfahren unabhängig von den entsprechenden kulturellen Paradigmen Pop-Ästhetik eigens bestimmen kann, bzw. wie sich das Verfahren allein dann von den Avantgarden unterscheiden sollte. Vielleicht muss die Frage überhaupt nicht gestellt werden, aber da es in der Bildenden Kunst und deren Erforschung durchaus so etwas wie eine poppige Ästhetik gibt, die als solche beschrieben worden ist, sollen die entsprechenden Vorschläge probeweise auf Literatur angewendet werden, um schließlich am Ende auch noch einmal auf die Avantgarde und den Ästhetizismus zu sprechen zu kommen.

Wenn unabhängig von Bezugnahmen auf Pop-Kultur gefragt werden soll, was an Texten pop-ästhetisch oder poppig sein kann, stiftet möglicherweise

50) Annette Dorgerloh, „Sie wollen wohl Ideale klauen...?“ Präfigurationen zu den Bildprägungen der „Neuen Frau“, in: Die Neue Frau. Herausforderungen für die Bildmedien der zoer Jahre, hrsg. von Katharina Sykora, S. 25-50, hier: S. 33; - Burcu Dogramaci, Mode-Körper. Zur Inszenierung von Weiblichkeit in Modegrafik und -fotografie der Weimarer Republik, in: Leibhaftige Moderne. Körper in Kunst und Massenmedien I9I8 bis 1933, hrsg. von Michael Cowan und Kai Marcel Sicks, Bielefeld 2005, S. I I9-I35, hier: S. I23.

${ }^{51}$ Bassler, Der deutsche Pop-Roman (zit. Anm. 9), S. I02. 
Richard Hamiltons Merkmalliste zu Pop Orientierung: ${ }^{52}$ ) popular, transient, expendable, low cost, mass produced, young, witty, sexy, gimmicky, glamorous, big business. Thomas Hecken schlägt folgende Definition vor, die einige Aspekte von Hamiltons Aufzählung im doppelten Wortsinn aufhebt: Oberflächlichkeit, Funktionalismus (Reiz, Attraktivität), Konsumorientierung, Äußerlichkeit, Immanenz, Künstlichkeit, Stilverbund. ${ }^{53}$ ) Oberflächlichkeit ist ein wesentliches Stichwort. „Pop ist nicht in der Tiefe eines Sinns verborgen und wartet dort auf Hermeneuten oder Dekonstruktivisten, auf Strukturalisten oder Systemtheoretiker - Pop gibt sich auf der Oberfläche zu erkennen. " ${ }^{45}$ ) Niels Werber verhandelt an dieser Stelle Oberflächlichkeit im Sinne einer Ereignisqualität eines Kunstwerks, die näher zu bestimmen wäre. Nebenbei bemerkt, wird Oberflächlichkeit von Werber als Aspekt des Gegenstandes aufgewiesen, aber implizit an der Art seiner Rezeption festgemacht. Es ist fraglich, ob man Pop pauschal etwas zuschreiben kann, was vielleicht eher Ausdruck eines Rezeptionsprozesses ist. Aber dennoch bildet Oberflächlichkeit, wie an dem Zitat zu sehen ist, ein zentrales Moment der Pop-Ästhetik. Es ist allerdings schwierig zu bestimmen, was dies bei Literatur konkret sein könnte.

Spektakuläre Selbstreferenz ist ein von Jochen Venus vorgeschlagenes und u. a. anhand eines Bildes von Donald Duck exemplifiziertes Konzept, mit dem Pop-Ästhetik in gewisser Hinsicht beschrieben werden kann. Ausgangspunkt ist die Annahme, dass beim populären Bild die „referentielle Ansicht [...] an die Stelle [rückt], die eigentlich die darstellerischen Mittel besetzen sollen. Sie wird Mittel zum Zweck." ${ }^{\text {(55) }}$ ) Hier geht es also gerade nicht um Referenzen. Der referentielle Bezugspunkt des Bildes ist nur noch ein Vorwand, Bildlichkeit als solche spektakulär zu inszenieren, oder anders gesagt: „Indem auf diese Weise das Donaldbild seine Ansichtsreferenz zum bloßen Gestaltungsmittel macht, weist seine Referenzialität auf die Bildlichkeit selbst zurück. Die Bildlichkeit wird selbstreferenziell. “56) Um keine Missverständnisse zu erzeugen, sei erwähnt, dass es im Folgenden nicht mehr um Jakobson geht, wenn von Fremdund Selbstreferenz die Rede ist.

Das Bild unterwirft alle potenziellen Betrachter demselben Sichtbarkeitsimperativ, so dass allen Betrachtern eines Bildes im Akt des Sehens evident wird, dass sie dasselbe sehen sollen. Und diese bestimmte Möglichkeit des Sehens, die durch ein konkretes Bild vorgeführt wird, kann entweder an vorhandene Sichtbarkeiten erinnern (ästhetische Referenz), nicht

52) Zitiert nach Hecken, Pop (zit. Anm. 6), S. ir. Die Liste wurde 1963 publiziert, nachdem Hamilton sie zuvor in einem Brief entworfen hatte.

53) Hecken, Pop-Konzepte der Gegenwart (zit. Anm. I5).

${ }^{54)}$ Werber, Szenarien der Ausnahme in der Populärkultur (zit. Anm. I2).

55) Venus, Die Erfahrung des Populären (zit. Anm. I5), S. 65.

${ }^{56)}$ Ebenda, S. 65. 
vorhandene Sichtbarkeit etablieren (ästhetische Referenzlosigkeit) oder bildlich etablierte Sichtbarkeiten als solche wiederholen (ästhetische Selbstreferenz). Dass das Bild eines Stuhls einen konkreten Stuhl intendiert - z. B. als Kaufangebot -, setzt kulturelles Wissen voraus. Aber dass ein Bild eine bestimmte Ansicht eines physischen Körpers zeigen kann, ist durch die Natur der menschlichen Wahrnehmung begründet, uns die Welt als eine körperliche Welt und uns als wahrnehmende Körper inmitten dieser körperlichen Welt zu zeigen..$^{57}$ )

Venus definiert Fremd- und Selbstreferenz im Hinblick auf visuelle Bilder. Da sein Vorschlag ziemlich interessant ist, soll seine ausblickende Frage, ob sich das Konzept auch auf literarische Verfahren übertragen ließe, $\left.{ }^{58}\right)$ analog zu Venus Argumentation eingehender erörtert werden. Wir haben es bei der Literatur mit einem Medium zu tun, das im Grunde inkommensurabel ist mit Kategorien wie Oberfläche, Spektakuläres oder Erleben, und das macht es so schwierig, von literarischem Pop zu sprechen. In der Literatur gibt es keinen verbindlichen Sichtbarkeitsimperativ, von dem man bei Bildern stets ausgehen kann und auf dessen Basis sich einstellen könnte, dass im Akt der Wahrnehmung unmittelbar das Gleiche erlebt wird.

Bei allen Vorbehalten kann aber doch davon ausgegangen werden, dass es möglicherweise literarische Äquivalente gibt, die Venus' Konzept entsprechen. In Keuns Romanen, insbesondere im `Kunstseidenen Mädchen`, wird recht plakativ mit diesen Aspekten umgegangen und dies poetisch im Sinne Jakobsons. Wenn Doris sagt: „Ich liebe Berlin mit einer Angst in den Knien“ (KM 9I) liegt eindeutig eine äquivalente kombinatorische Selektion vor, weil Angst sprichwörtlich zwar in den Knien verortet wird, es aber auf dem Reim basiert, dass dieses und kein anderes Sprichwort verwendet wird.9) Ein Binnenreim ist erwünscht, ebenso der Knittelvers. Den Stilmitteln, mehr aber noch dem eigentlichen Sprichwort würde man auch mit dem Satz ,Ich liebe Berlin mit zitternden Knien' Genüge tun, aber offenbar soll ebenso die Angst plakativ benannt werden, weshalb es hier zu einer Übertreibung und Doppelung kommt. Dieser Satz in einem Prosatext bezieht sich recht auffällig auf Stilmittel der Lyrik, dies umso mehr, als Johann Wolfgang von Goethe zu Beginn des Romans erwähnt wird, der neben vielem auch für seine Knittelverse bekannt ist.

Der Text ist hier sinnlich, sinnlos, übertrieben, verspielt, selbstreferentiell. Ähnlich wirken die Alliterationen in folgendem Satz: „Ich sammle Sehen für ihn. Ich gucke mir alle Straßen an und Lokale und Leute und Laternen. Und dann merke ich mir mein Sehen und bringe es ihm mit" (KM 93). Neben der Häufung des $S$ folgt die Selektion aus dem Paradigma Straßenatmosphäre dem

57) Ebenda, S. 6I.

${ }^{58}$ ) Ebenda, S. 68

59) Arend und Martin, Irmgard Keun (zit. Anm. 4), S. 260-264. 
phonologischen Paradigma L. Es mag überzogen sein, die wenigen ähnlichen Momente in Krachts Roman zu fokussieren, aber eine Alliteration ist besonders auffällig. Nach allen aversiven Ausführungen bezüglich seiner Umgebung äußert der Erzähler den Satz: „Links und rechts der Straße rast Sylt an uns vorbei, und ich denke: Sylt ist eigentlich super schön" (FL I I). Im Gegensatz zu dem K in dem Satz auf der gleichen Seite „Kurz vor Kampen biegt Karin plötzlich ab [...]" fällt die Häufung der $S$ deshalb so besonders auf, weil hier der Einbruch einer authentischen positiven Empfindung inszeniert wird. Aussage und Form verweisen aufeinander, sodass wir es mit einer zentralen Stelle zu tun haben, in der ein idyllischer Moment aufscheinen soll. Im Grunde handelt es sich bei dem Roman ja um eine Sinnsuche. Darin unterschiedet sich Kracht einmal mehr von Keun, weil dieser Stilmittel als Mittel einsetzt, während sie bei Keun einen oberflächigen, ästhetischen Selbstzweck bilden.

Neben den aisthetischen Momenten simuliert Keuns zweiter Roman in intermedialen Verfahren Spektakularität. Er spielt systematisch mit fremdmedialen Möglichkeiten, so auch dem Kinofilm.

Und da muß ich doch viele Farben gesehen haben: „Ich habe gesehen - [...] Plakate mit nackten rosa Mädchen [...]. Ich habe gesehen - ein Mann mit einem Plakat um den Hals: ,Ich nehme jede Arbeit - und 'jeder dreimal rot unterstrichen - es gab eine Frau ihm zehn Pfennig, die waren gelb, und er rollte sie auf das Pflaster, was Schein hat durch Reklame von Kinos und so Lokalen. Und das Plakat war weiß mit schwarz drauf. Und viele Zeitungen und sehr bunt und das Tempo rosa-lila und Nachtausgabe mit rotem Strich und ein gelber Querschnitt - und sehe das Kempinski [...]. Ich sehe - gequirlte Lichter [...]. (KM 97-101)

Was Doris sieht, sieht der Leser oder die Leserin ebenso wenig wie der blinde Brenner, d.h. das Anliegen einer Romanpassage, in der einem Blinden visuelle Eindrücke vermittelt werden sollen, ist sehr offenkundig als reflexiv poetologisches zu verstehen. Die Herausforderung besteht darin, sprachlich Visualität zu simulieren und sich am neuen Medium Kino zu erproben, also Spektakularität zu erzeugen. Schauspielerinnen, Filmhandlungen etc. spielen ohnehin eine große Rolle im 'Kunstseidenen Mädchen.$\left.^{60}\right)$ Hier geht es nun um den filmischen Stil. ${ }^{61}$ ) Die Gedankenstriche simulieren keine Pausen, sondern die Kameraschnitte zwischen wechselnden Blickpunkten in einem Film. Rosa, rot, gelb, bunt, rosa-lila, rot, gelb bleiben zwar sprachliche Zeichen, verweisen aber in ihrer Häufung wohl auf den Mangel an Farbe in der Sprache.

Der Kinofilm war in den $20 e r$ Jahren schwarz-weiß. Zwei Jahre vor Erscheinen des Romans hatte die UFA allerdings ihren ersten Farbfilm gezeigt. Das

${ }^{60}$ ) Leo A. Lensing, Cinema, Society, and Literature in Irmgard Keun's Das kunstseidene Mädchen, in: The Germanic Review 60 (I985), Nr. 4, S. I29-I34, hier: S. I32-I33.

${ }^{61}$ ) Irina Rajewsky, Intermedialität (= Uni Taschenbücher M), Tübingen 2002, S. 8. 
Thema Farben stellt eine intermedial wichtige Kategorie zu der Zeit dar. Licht spielt ebenfalls eine Rolle in der Textpassage, was sowohl auf Berlin als auf die Grundbedingung von Kinofilmen verweist. Plakate, Reklame, Kinos, Zeitungen werden als fremde Medien benannt. Konkreter wird auf die Zeitschriften ¡Tempo und 'Querschnitt Bezug genommen. Die Begriffe beschreiben aber außerdem in einem poetologischen Sinn den Stil der Passage, der abgehackt, dynamisch und atemlos ist. Selbstreferentiell ist die Passage überdies auch, weil sie auf die eigenen Konstruktionsbedingungen verweist: Es wird mit dem Vermögen und Unvermögen des eigenen Mediums im Vergleich mit anderen gespielt.

Krachts Roman spielt im Vergleich mit Keun wenig auf den Film an. Die bereits zitierte Badewannen-Szene ist jedoch nicht nur unangenehm plastisch, sondern liefert mit dem Verweis auf den Film `Der Exorzist ein Ausgangsbild, wie man sich das Erbrechen vorzustellen hat. Die Nahrungsmittelreste werden außerdem sehr lebendig, obwohl es sich letztlich um eine knappe Textstelle handelt, die stilistisch nicht zur Ekphrasis tendiert. Da Erbrochenes einen starken olfaktorischen Reiz darstellt, reicht der Begriff „Klümpchen“, um Übelkeit zu evozieren. Die Verfahren kommen hier aber ohne Selektion aus dem Paradigma der Pop-Kultur aus. Keuns Texte mehr, Krachts Text weniger weisen in gewisser Weise sprachgenüssliche, verspielte, aisthetisch effektheischende Momente auf, man käme aber nie auf die Idee, sie als literarischen Pop zu beschreiben, wenn es keine Referenzen auf die Pop-Kultur gäbe.

Jedoch auch hinsichtlich der Verankerung dieser Referenzen unterscheiden sich Keuns und Krachts Romane. Bei Keun findet nämlich eine Verkehrung von Zweck und Mittel in Venus' Sinn statt, bei der die Fremdreferenz im Dienste der Selbstreferenz wieder ins Spiel kommt. Bedenkt man das Verhältnis von origineller Geschichte und Collage-Elementen, kann durchaus behauptet werden, dass die Geschichten von Keuns Romanen nur den Anlass bilden, Fremdes, also massenmedial Vermitteltes, Populäres und Pop-Kultur zur Geltung zu bringen, nicht umkehrt. Es findet in den Texten also eine Umkehrung dessen statt, was traditionell als Zweck und Mittel des Erzählens verstanden wird. Und das Fremdreferentielle ist letztlich das Selbstreferentielle, weil es sehr klare Markierungen des eigenen Verfahrens gibt, die als solche in den Blick rücken, denn es handelt sich um offene Collage-Verfahren. ${ }^{62}$ ) Mit Keuns Texten liegen

${ }^{62}$ ) In Collage- und Intermedialitätstheorien wird stets darauf verwiesen, dass es einen entscheidenden Unterschied macht, ob die entsprechenden Verfahren offen oder verdeckt zur Anwendung kommen. (Volker Hage, Einleitung, in: Literarische Collagen. Texte, Quellen, Theorien, hrsg. von Volker Hage, Stuttgart i98I, S. 5-30, S. i i; - Volker Klotz, Zitat und Montage in neuerer Literatur und Kunst, in: Literatur und Bildende Kunst. Ein Handbuch zur Theorie und Praxis eines komparatistischen Grenzgebiets, hrsg. von UlRICH Weisstein, Berlin 1992, S. I80-195, hier: 186-187; - RAJEwsky, Intermedialität, zit. Anm. 6I, S. 82). 
keine Romane vor, die Pop-Kultur einfach inkarnieren, sondern Romane, die ihre eigene Ästhetik im Angesicht der Pop-Kultur reflektieren und ostentativ zur Schau stellen.

Es geht nicht um die Ästhetik der Pop-Kultur, sondern um die Ästhetik der Collage. Das heißt, das Pop-Kulturelle trägt zur Selbstreferenz des Textes bei, während das Epische, das traditionell in Prosatexten im Vordergrund steht, überspitzt formuliert, zu einem Transportmittel ,degradiert' wird. In Bezug auf Krachts Text trifft das nicht im gleichen $\mathrm{Maß} z u$, weshalb er letztlich viel weniger als Pop-Roman klassifiziert werden kann als Keuns Romane. Hier scheint die Reisegeschichte, ob man sie nun als Flucht oder Suche bezeichnen soll, doch deutlich im Zentrum zu stehen, das Epische also den Zweck des Erzählens zu bilden, während die eingelassenen Verweise entweder nur Ornat, also Mittel im Dienste der Geschichte bilden, oder zu dieser selbst gehören, weil sich der Erzähler einen Weg aus ihnen heraus bahnen will. Die Referenzen werden im Grunde nie auf einer ästhetischen Oberfläche um ihrer selbst willen sichtbar, sondern haben eine Funktion im Syntagma bzw. sind ins Syntagma eingebunden.

Wenn man die mehr oder weniger avantgardistischen Verfahren der Textentgrenzung in Richtung Pop-Kultur mit den ästhetizistischen Verfahren zusammen bedenkt, ergibt sich möglicherweise so etwas wie eine Pop-Ästhetik. Letztlich stellt insbesondere Keuns, Glanz' eine reflexive Metapher dar, mit der sie um eine Ausdrucksmöglichkeit für ein Verfahren ringt, das ab den soer Jahren möglicherweise als Pop bezeichnet worden wäre, für das es in den zoer Jahren aber noch keinen Begriff gab! Ästhetik, Konsum, Hedonismus, ,Attitü$\mathrm{de}^{\mathrm{r}_{3}}$ ) und Distinktion stehen gleichzeitig im Zeichen von Populärkultur und Kulturindustrie und im Zeichen des Ästhetizismus, ${ }^{64}$ ) was zusammen letztlich Pop ergibt. Zunächst einmal wird eine Entgrenzung von Kunst betrieben, die an den Avantgardismus erinnert. Im Avantgardismus geht das Konzept aber nicht ganz auf, weil zusätzlich zur Entgrenzung auch eine Übersteigerung von Kunst vorgeführt wird. Wie Populärkultur zur l'art pour l'artwerden kann, und l'art pour l'art keinen Widerspruch zur realitätszugewandten oder hyperrealen Alltagskunst darstellt ${ }^{65}$ ) zeigt sich in dieser paradoxen Konstellation. „Avantgardistische Kulturformen, die das Präfix Pop tragen, affirmieren den Pop auf ästhetizistische Weise. Während sich der alte Ästhetizismus einer sakralen

${ }^{63}$ ) Schumacher und Gleba: Vorwort, in: Schumacher und Gleba, Pop seit ig64 (zit. Anm. 9), S. I I-I6, hier: S. I2.

${ }^{64)}$ Ingo Niermann, Oberfläche, in: Grabienski, Huber und Thon, Poetik der Oberfläche (zit. Anm. II), S. 227-230, hier: S. 227.

${ }^{65}$ ) Markus Tillmann, Populäre Musik und Pop-Literatur. Zur Intermedialität literarischer und musikalischer Produktionsästhetik in der deutschsprachigen Gegenwartsliteratur, Bielefeld 2013, S. 74. 
Herrschaftskultur annahm, widmet sich dieser Pop zweiter Ordnung einer Oberfläche, die nicht mehr zu sein beansprucht als banal.“"66)

Das heißt, wir haben es mit einem Ästhetizismus zu tun, der bereits eine avantgardistische Entdifferenzierung voraussetzt. Die ästhetischen Formen stammen nämlich aus den Massenmedien und der Pop-Kultur der Weimarer Republik. Die Wände zu dieser werden aber nicht eingerissen, sondern die Tür ist längst geöffnet. Entdifferenzierung muss nicht von Künstlern betrieben werden - das war eine vielleicht naive und ohnehin nicht konsequent realisierte Haltung der Avantgardisten - sondern Keuns Romane zeigen, dass ein Teil der Medienrealität der Weimarer Republik längst eine eigene Sphäre zwischen Fakt und Fiktion, zwischen Konsum und Ästhetik darstellt, die die Romane als Resonanzräume nur aufzugreifen und zu reflektieren brauchen. ${ }^{67}$ )

Damit demokratisieren sich die Texte nicht, sondern sie pflegen ihren eigenen, neuartigen Elitarismus, was sich z. B. in der Vokabel ,Glanz zeigt, einem Begriff mit sehr vager Semantik, der aber den gesamten Text strukturiert. ${ }^{68}$ )

Ich will so ein Glanz werden, der oben ist. Mit weißem Auto und Badewasser, das nach Parfüm riecht, und alles wie Paris. Und die Leute achten mich hoch, weil ich ein Glanz bin und werden es dann ganz wunderbar finden, wenn ich nicht weiß, was eine Kapazität ist und nicht runter lachen auf mich wie heute [...]. Ich werde ein Glanz, und was ich dann mache, ist richtig - nie mehr brauch ich mich in acht nehmen und nicht mehr meine Worte ausrechnen und meine Vorhabungen ausrechnen - einfach betrunken sein - nichts kann mir mehr passieren an Verlust und Verachtung, denn ich bin ein Glanz. (KM 44/45)

Da Glanz mit rauschhaften Vorstellungen von Glamour, wie sie Hollywoodfilme, illustrierte Zeitschriften und auch Schlager verbreitet haben, verbunden wird, bezieht sich der Roman hier auch auf Populärkultur und Kulturindustrie. Liest man die Textstelle poetologisch, wird aber auch deutlich, dass Glanz als Ausdruck für eine lustvolle, ästhetische Selbstermächtigung verwendet wird natürlich auch für eine psychologische. Der Begriff erinnert an ein ästhetizistisches Konzept, das in Oscar Wildes `Dorian Gray` als „radiance“ bezeichnet wird, ${ }^{69}$ ) und das die sehr artifizielle und spielerische Anlage des $/$ Kunstseidenen

${ }^{66}$ ) Niermann, Oberfläche (zit. Anm. 64), S. 228.

${ }^{67}$ ) Lickhardt, Irmgard Keuns Romane (zit. Anm. 6), Kap. 4.

${ }^{68)}$ Gudrun Raff, Leben. Szenen eines Täuschungsspiels. Zu literarischen Techniken Irmgard Keuns, Diss. Hamburg 2000, S. 76; - Ariane Martin, Kultur der Oberfläche, Glanz der Moderne. Irmgard Keuns Das kunstseidene Mädchen, in: Deutschsprachige Romane der klassischen Moderne, hrsg. von Matthias Luserke-JaQui, S. 349-367, hier: S. 354.

${ }^{69}$ ) Maren Lickhardt, Joe Lederer und Irmgard Keun. Glück als Ästhetik der Oberfläche und Vergnügen bei der Lektüre in Romanen der Weimarer Republik, in: Glück paradox. Moderne Literatur und Medienkultur, theoretisch gelesen, hrsg. von AnJa GerigK, Bielefeld 2010, S. 153-182, hier: S. 166. Für die Pop-Literatur der 90er Jahre konstatiert Niels Werber, sie sei mit ästhetizistischer Literatur vergleichbar (Niels Werber, „Das graue Tuch der Langeweile“, zit. Anm. 45). Insbesondere gilt dies für die Wildesche Ausprägung: 
Mädchens bezeichnet, das „Kunstprosa“70) ist, also eine glanzvolle Oberflächenästhetik mit starker Hyperbolik, Melodramatik und Sinnlichkeit an den Tag legt. Das bezeichnet den herausgehobenen Modus von Keuns Romanen. Schließlich hebt der Glanz Doris wie den Text aus der Umgebung heraus. Doris ist bezeichnenderweise "ganz verschieden von Therese und den anderen Mädchen auf dem Büro und so, in denen nie Großartiges vorgeht" (KM Io). Der Glanz ist Mittel der Distanzierung: „Und sie [Frauen, die ein ,Glanz sind] sind ihre eigne Umgebung und knipsen sich an wie elektrische Birnen, niemand kann ran an sie durch die Strahlen" (KM 79). Das elektrische Licht, nicht Sonne oder Sterne erzeugen den Glanz. Es darf nicht übersehen werden, dass die Strahlen sehr wohl blenden können. Jedoch ist vor allem interessant, dass in dem Bild sowohl die psychologische Immunisierung und Panzerung anklingen, die den Blick auf das Innere verhindern, als auch die ästhetische Erhabenheit, die das Kunstwerk abdichtet und exklusiv macht.

Es ist sehr schwer, Keun literaturgeschichtlich einzuordnen, wenn man allen textuellen Paradoxien gerecht werden will. Im Begriff des Pop scheinen aber erstaunlicherweise viele Phänomene aufgehoben zu sein, die Keuns Romane auszeichnen: ein poetologisches Programm der Enthierarchisierung und Entgrenzung von Kunst, ein poetologisches Programm der Übertreibung künstlerischer Mittel, Aufwertung der kulturindustriellen und pop-kulturellen Bedingungen, oberflächliche, gleichermaßen selbst- wie fremdreferentielle Verfahren und vor allem eine enorme Drastik, Hyperbolik und Ausgelassenheit. Vielleicht zeigen Keuns Texte, dass das Zusammentreffen von Entgrenzung und Übertreibung von Kunst eine poppige Angelegenheit ist, die in der Weimarer Republik erstmals beobachtet werden kann. Allgemeiner formuliert, wird u.a. mit Keuns Romanen die avantgardistische Entdifferenzierung von Kunst wieder in das Kunstsystem aufgenommen und als Kunstform, und zwar als Pop-Ästhetik, ausdifferenziert. Tatsächlich trifft vieles der genannten Merkmale auch auf Kracht zu, aber eben auf dessen Selbstinszenierung als Autor und weniger auf den Roman 'Faserland a als Text/Textur, denn dieser begibt sich zwischen der Nordsee und einem Schweizersee auf die Suche nach der verlorenen Idylle.

\footnotetext{
„Mit Wilde tritt der Dandy in das Zeitalter der Massenkultur ein“ (Günter Erbe, Der moderne Dandy. Zur Herkunft einer dekadenten Figur, in Tacke und Weyand, Depressive Dandys, zit. Anm. Io, S. 17-38, hier: S. 35).

${ }^{70}$ ) Arend und Martin, Irmgard Keun (zit. Anm. 4), S. 256.
} 
